\title{
Indirect observation of phase conjugate magnons from non-degenerate four-wave mixing
}

\author{
Alistair Inglis ${ }^{1}$ (D) . Calvin J. Tock ${ }^{1}$ (D) John F. Gregg ${ }^{1}$
}

C The Author(s) 2019 OPEN

\begin{abstract}
A phase conjugate mirror utilising four-wave mixing in a magnetic system is experimentally realised for the first time. Indirect evidence of continuous-wave phase conjugation has been observed experimentally and is supported by simulations. The experiment utilises a pump-probe method to excite a four-wave mixing process. Two antennae are used to pump a region of a thin-film yttrium iron garnet waveguide with magnons of frequency $f_{1}$ to create a spatio-temporally periodic potential. As the probe magnons of $f_{\mathrm{p}}$ impinge on the pumped region, a signal with frequency $f_{\mathrm{c}}=2 f_{1}-f_{\mathrm{p}}$ is observed. The amplitude of the nonlinear signal was highly dependent on the applied magnetic field $H$. Width modes of the probe magnons and standing wave modes of the pump magnons were shown to affect the amplitude of the signal at $f_{\mathrm{c}}$. Experimental data are compared with simulations and theory to suggest that $f_{\mathrm{c}}$ is a phase conjugate of $f_{\mathrm{p}}$.
\end{abstract}

Keywords Magnons $\cdot$ Spin waves $\cdot$ Phase conjugate $\cdot$ Four-wave mixing

\section{Introduction}

The insatiable appetite for smaller, more powerful computing devices is leading to the inevitable breakdown of Moore's Law [29]. A considerable deviation from Dennard's scaling is already underway [18] opening the door for alternative computational paradigms. In recent years, there has been increasing interest in magnon-based computing, or magnonics, $[5,7,8,11,12,24,26]$ as a solution to particular problems [34] facing the future of conventional complementary metal-oxide-semiconductor (CMOS) computing.

An advantage of wave computing not restricted to magnonics is the ability to encode information in two variables: amplitude and phase. The ability to perform operations on these parameters is therefore of fundamental importance to the future of wave computing. The study of nonlinear systems [1] opens the door for the development of these novel operations. One useful operation, in particular, is phase conjugation; a process that exactly reverses the propagation direction and phase factor for every plane wave in an arbitrary wavefront [10]. The result is the creation of a phase conjugate mirror (PCM) that reflects any beam along the same path by which it arrived at the mirror, irrespective of incident angle. This remarkable property can lead to aberration correction of waves after passing through a nonuniform distorting medium; a process with many useful applications including image processing, encryption and spectroscopy $[4,9,27]$.

First experimentally realised in the 1970s, phase conjugate mirrors are a well-established phenomenon in the optical community. A preferred method for creating a PCM is by way of the third-order nonlinear process: four-wave mixing (FWM)[2]. Experiments of this nature have a general form wherein the confluence of two 'pump' beams and a'probe' beam in a region of nonlinear medium causes the appearance of a fourth beam which is the phase conjugate of the probe signal [3]. In addition to creating a PCM, this method of optical FWM has lead to a number of novel physical processes, including squeezing [17], vortices [32],

Alistair Inglis, alistair.inglis@physics.ox.ac.uk| ${ }^{1}$ Clarendon Laboratory, Department of Physics, University of Oxford, Parks Road, Oxford OX1 3PU, UK.

SN Applied Sciences (2019) 1:480 | https://doi.org/10.1007/s42452-019-0500-x 
and the emergence of upto fifth-order nonlinear effects [30].

Until now, phase conjugation of spin waves has only been achieved using methods of parametric pumping and second-order nonlinear processes $[20,21,25]$. In this work, we report on the creation of a PCM in a magnon waveguide. Our experiments differ from previous work for three reasons: (1) We achieve a phase conjugate with the process of FWM, rather than parametric pumping. (2) Our experiments observe a phase conjugate using the continuous-wave (CW) regime, in contrast to the pulsed experiments that utilise spin wave bullets. (3) In contrast to similar optical experiments [33], we exploit the large magnon beam widths, the strength of the nonlinear interaction and the isotropic magnon dispersion relation to implement a geometry that has orthogonal pump and probe beams.

In general, for FWM to occur certain physical conditions must be met. Energy conservation dictates that

$\omega_{1}+\omega_{2}=\omega_{\mathrm{p}}+\omega_{\mathrm{c}^{\prime}}$

where $\omega_{1,2}$ are the angular frequencies of the two pump waves, $\omega_{\mathrm{p}}$ is the probe wave angular frequency, and $\omega_{c}$ is the conjugate wave angular frequency. Similarly, the condition

$\mathbf{k}_{1}+\mathbf{k}_{2}=\mathbf{k}_{\mathrm{p}}+\mathbf{k}_{\mathrm{c}^{\prime}}$

arises from momentum conservation, where $\mathbf{k}$ is a magnon wave vector and the subscripts follow the same convention as the angular frequencies. These constraints are met by wave vectors depicted by Fig. $1 \mathrm{~b}$. It is clear that if $\mathbf{k}_{1}$ and $\mathbf{k}_{2}$ serve as counter-propagating pumps of same frequency in an isotropic medium and $\mathbf{k}_{\mathrm{p}}$ serves as the probe then by equation (2), the condition $\mathbf{k}_{\mathrm{p}}=-\mathbf{k}_{\mathrm{c}}$ must be satisfied.

We now consider FWM in a nonlinear magnetic system. By examining the Landau-Lifshitz equation with a perturbation expansion of the magnetisation, it has been shown

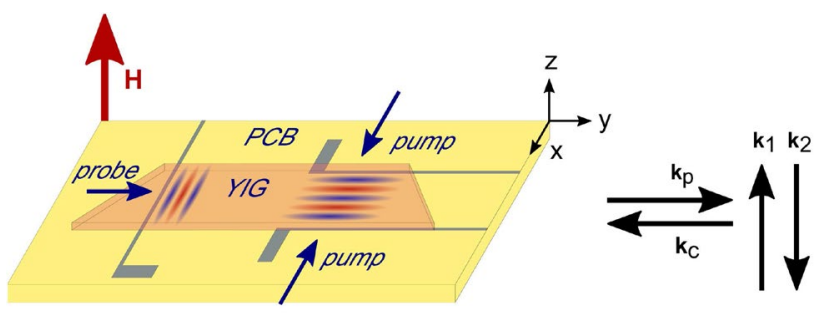

(a)

(b)

Fig. 1 a Experimental configuration: yttrium iron garnet (YIG) film with $45^{\circ}$ edges placed on PCB with antennae. Counter-propagating pumps excite a standing wave which creates a periodic potential. The probe antenna transmits excitation magnons and receives reflections from the pumped region. $\mathbf{b}$ Illustration of the conservation of momentum condition for four-wave mixing where $\mathbf{k}_{1}$ and $\mathbf{k}_{2}$ are the pumps, $\mathbf{k}_{\mathrm{p}}$ is the probe, and $\mathbf{k}_{\mathrm{c}}$ is the phase conjugate that there exists a driving term for a third-order spin wave that depends on the product of $m^{3}[16,19]$, where $m$ is a component of the transverse magnetisation.

To understand the origin of the expected phase conjugate magnon, consider the $\mathrm{m}^{3}$ term in the location of the pumped region with the probe magnons also present. This term may be expanded into its constituent parts. Following a mathematics analogous to the derivation of optical phase conjugation [31], we expand $m^{3}$ and express it as a real quantity:

$$
\begin{aligned}
& \left(m_{1} e^{i\left(\omega_{1} t-\mathbf{k}_{1} \cdot \mathbf{r}\right)}+m_{1}^{*} e^{-i\left(\omega_{1} t-\mathbf{k}_{1} \cdot \mathbf{r}\right)}\right. \\
& \quad+m_{2} e^{i\left(\omega_{2} t-\mathbf{k}_{2} \cdot \mathbf{r}\right)}+m_{2}^{*} e^{-i\left(\omega_{2} t-\mathbf{k}_{2} \cdot \mathbf{r}\right)} \\
& \left.\quad+m_{\mathrm{p}} e^{i\left(\omega_{\mathrm{p}} t-\mathbf{k}_{\mathrm{p}} \cdot \mathbf{r}\right)}+m_{\mathrm{p}}^{*} e^{-i\left(\omega_{\mathrm{p}} t-\mathbf{k}_{\mathrm{p}} \cdot \mathbf{r}\right)}\right)^{3} .
\end{aligned}
$$

Here $m_{1,2}$ and $m_{p}$ represent the amplitude of the transverse magnetisation of the pumps and probe, respectively. Since $m_{i}$ is a complex amplitude, it also contains the phase information. Upon expansion of equation (3), we obtain 56 cross-terms. Terms with phase factors that have combinations of $\omega$ and $\mathbf{k}$ that are forbidden by the dispersion relation may be neglected. Of the remaining terms, there is one of particular significance:

$$
\begin{aligned}
& m_{1} m_{2} m_{\mathrm{p}}^{*} e^{i\left(\left[\omega_{1}+\omega_{2}-\omega_{\mathrm{p}}\right] t-\left[\mathbf{k}_{1}+\mathbf{k}_{2}-\mathbf{k}_{\mathrm{p}}\right] \cdot \mathbf{r}\right)}+\text { c.c. } \\
& \quad=m_{1} m_{2} m_{\mathrm{p}}^{*} e^{i\left(\omega_{c} t-\mathbf{k}_{c} \cdot \mathbf{r}\right)}+\text { c.c.. }
\end{aligned}
$$

There are a number of points to note about this term. Firstly, it is proportional to $m_{\mathrm{p}}^{*}$ and is therefore the phase conjugate of $m_{\mathrm{p}}$. Secondly, this is the only possible term possessing a wavevector antiparallel to the original probe beam $[22,31]$. These two properties define the resulting spin wave as a phase conjugate reflection. Finally, we note that this term is proportional to $m_{1}^{2}$ assuming the pumps are of equal magnitude, that is $m_{1}=m_{2}$.

\section{Experiment}

For the magnon waveguide, we utilised a yttrium iron garnet (YIG) film of thickness $7.8 \mu \mathrm{m}$ on a gallium gadolinium garnet (GGG) substrate, $2.1 \mathrm{~mm}$ wide and $18 \mathrm{~mm}$ long, with corners cut at $45^{\circ}$ to minimise reflections. A schematic of the set-up is shown in Fig. 1a. The waveguide was mounted on a printed circuit board (PCB) with three antennae. The antennae were in the formation of a meander structure to suppress the coupling to the ferromagnetic resonance (FMR) mode. The antennae were $4 \mathrm{~mm}$ long comprising three legs $40 \mu \mathrm{m}$ wide, spaced $50 \mu \mathrm{m}$ apart. The two pump antennae were parallel to the long edge

\section{SN Applied Sciences}


of the waveguide. The probe antenna was placed $3 \mathrm{~mm}$ from the pumps, across the width as shown in Fig. 1a. The probe antenna is used for both transmission and detection of magnons interacting with the pumped region, while the pump antennae excite counter-propagating spin waves that exploit the strong intrinsic nonlinearity of the magnon system to generate a periodic mesoscopic texture.

In our experiment, spin waves travelling in both the $x$ and $y$ directions were utilised. For this reason, an isotropic magnon dispersion was required. Such a condition is offered by exciting forward volume magnetostatic spin waves (FVMSWs) which have a uniquely isotropic dispersion, compared to the highly anisoptropic dispersion relations for other magnetostatic spin wave modes [23]. An electromagnet was used to apply the external magnetic field perpendicular to the plane of the film, a field geometry that is necessary for the excitation of FVMSWs.

The pump antennae were excited at $f_{1}=f_{2}=3.915 \mathrm{GHz}$ by a Hewlett-Packard HP8672A microwave source. A second source (HP8671A) was used to excite the probe antenna at $f_{\mathrm{p}}=3.91825 \mathrm{GHz}$. This detuning $\Delta f=3.25 \mathrm{MHz}$ was required to use the spectrum analyser to discriminate between input and output signals. Introducing $\Delta f$ and ensuring Equations (1) \& (2) are satisfied leads to a small phase mismatch $\Delta k$ which manifests itself experimentally as a reduced efficiency [10, 22].

The power of the probe magnons immediately after insertion was approximately $8 \mu \mathrm{W}$, which attenuated to approximately $2 \mu \mathrm{W}$ after propagation towards the pumped region, which was populated by spin waves of power approximately $40 \mu \mathrm{W}$. The combined spin wave power is above the reported nonlinear threshold for systems satisfying the Lighthill criteria $[13,15]$ as ours does.

The circulator shown in Fig. 2 allows the probe to act as a transmitter-receiver antenna. Reflections from the

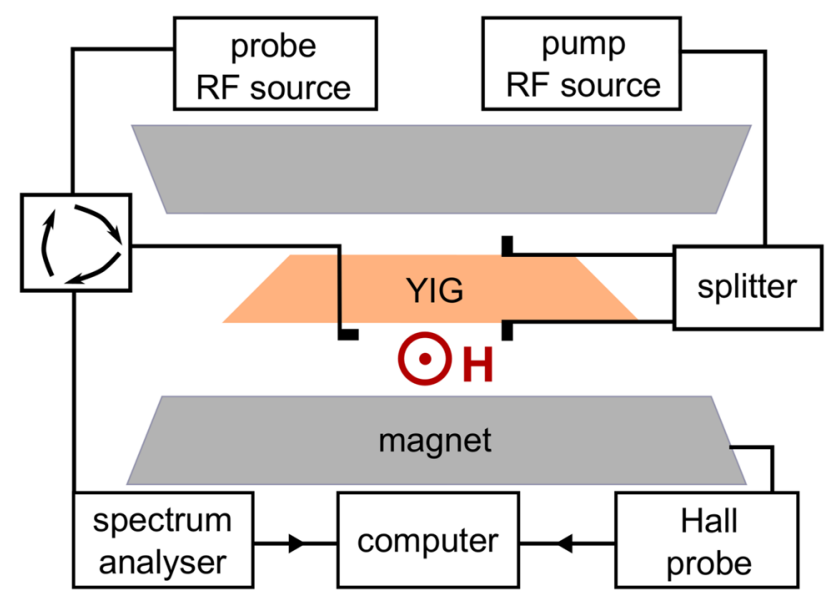

Fig. 2 Schematic of experimental set-up. Magnetic field is perpendicular to the plane of the YIG waveguide pumped region will propagate back towards the probe antenna and be detected by the spectrum analyser ( $\mathrm{ZHL}$ Rhode \& Schwarz). The magnetic field was measured with a Hall probe which was connected to a data acquisition computer.

\section{Results and discussion}

Figure $3 a$, $b$ shows the measured spectra for two different field configurations. When there is no external field applied as in (a), the only signals measured are the input signals. The large signal at $f_{\mathrm{p}}$ is due to the impedance mismatch between the microwave transmission line and the probe antenna causing electrical reflections to be detected by the spectrum analyser, while the peak at $f_{1}$ is due to direct coupling from the pump antennae to the probe antenna. As the magnetic field was increased to 3077 Oe,
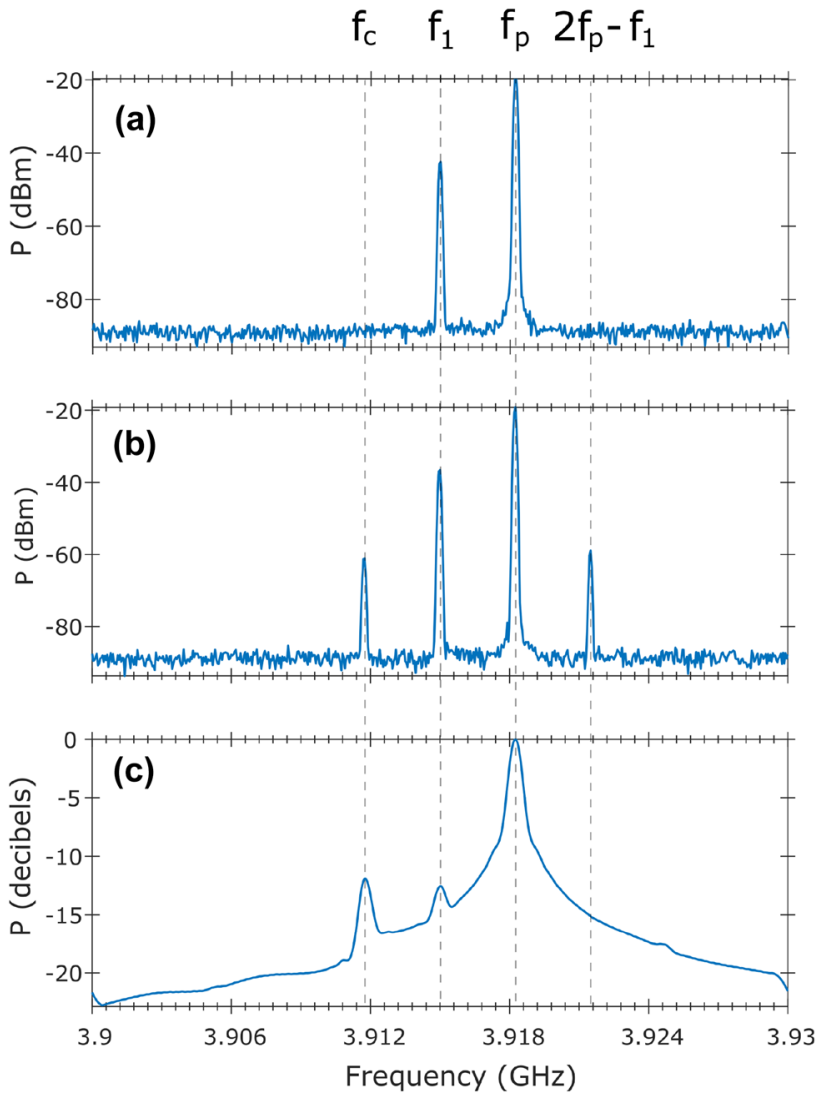

Fig. 3 Measured and calculated frequency spectra. a Spectrum measured with no magnetic field applied. Both peaks are artefacts of the experimental electronics. b Spectrum measured with applied magnetic field of 3077 Oe. c Spectrum from simulated pump-probe system at 3077 Oe. The large width of the peak at $f_{\mathrm{p}}$ is due to limited computational resolution and conversion to a logarithmic scale. Both measured and calculated spectra show peaks at expected phase conjugate frequency $f_{c}$ 


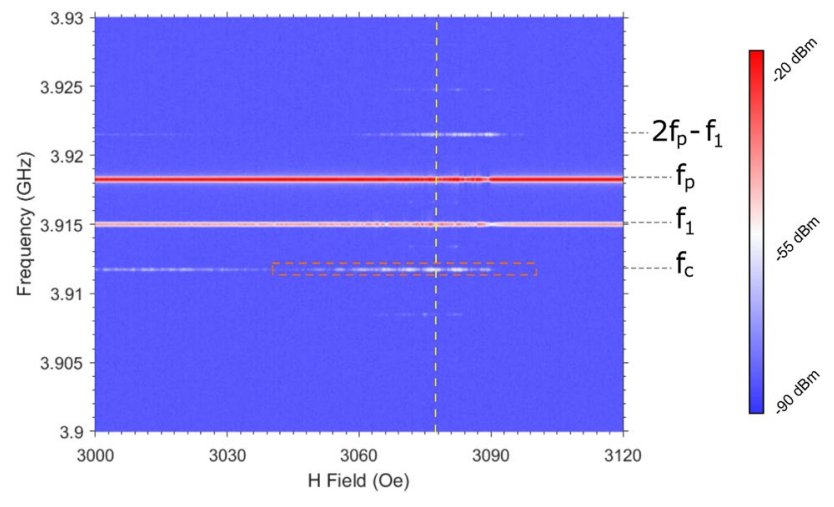

Fig. 4 Output of probe antenna as a function of magnetic field and frequency. Dashed yellow line corresponds to spectrum in Fig. $3 \mathrm{~b}$. Orange boxed region highlights signal at $f_{c}$. The magnetic field dependence of boxed region is shown in more detail in Fig. 5

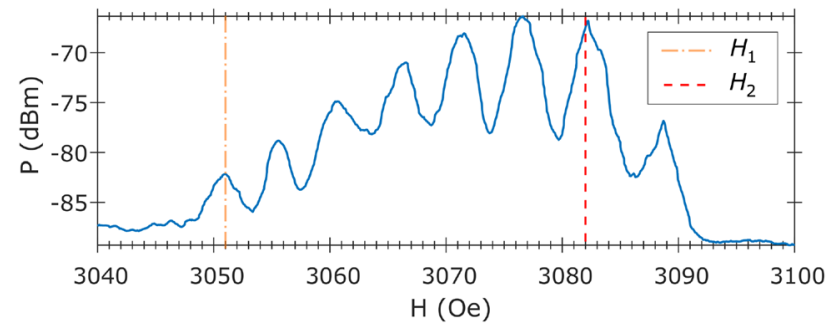

Fig. 5 Magnetic field dependence of the $f_{\mathrm{c}}=3.91175 \mathrm{GHz}$ signal returning to the probe antenna. Fast oscillation shows the dependence on the standing wave mode of the pump. The lower amplitude at $H_{1}=3051$ Oe compared to $H_{2}=3082$ Oe is attributed to width modes, simulations of which are shown in Fig. 6

the spectrum $3 \mathrm{~b}$ was observed. Of note is the signal at the expected phase conjugate frequency $f_{\mathrm{c}}=2 f_{1}-f_{\mathrm{p}}$. This is not inconsistent with the notion that at the correct field strength, phase conjugate reflections are occurring from the pumped region. Also present in the spectrum is a term at frequency $f=2 f_{\mathrm{p}}-f_{1}$ which is due to a third-order process resulting from reflections of probe magnons from the waveguide edge farthest from the probe antenna.

Investigating further, a sweep of the applied magnetic field was performed, the results of which are shown in Fig. 4. The dashed yellow line marks the field at which the spectrum in Fig. 3b was measured. The strong lines at $f_{\mathrm{p}}$ and $f_{1}$ appear to have minimal field dependence since the field dependent contribution to the signal is small compared to the electrical response described above.

More interesting, however, is the white line at $f_{c}=3.91175 \mathrm{GHz}$ showing an obvious field dependence. The nature of the dependence of the boxed region can be seen in more detail in Fig. 5. The amplitude reaches the noise floor at a field of approximately 3093 Oe. This is due to the excited FVMSWs approaching the FMR above which no more spin waves are excited. Below this field however, the signal oscillates as a function of field, with a period of approximately $5 \mathrm{Oe}$. This oscillation corresponds to different standing wave modes across the width of the pumped region. That is, when there are an integer number of halfwavelengths across the width of the waveguide, a standing wave is present and the pump amplitude is larger. This in turn, amplifies the phase conjugate signal, since it scales with $m_{1} m_{2}$ as shown in equation (4).

In addition to this fast oscillation, Fig. 5 also shows a field dependence on a larger scale. This may be explained by considering width modes [14] of the probe magnons. At approximately 3040 Oe, the width modes excited by the probe antenna destructively interfere, inhibiting the propagation of the probe spin wave, thus diminishing the interaction with the pumped region.

\section{Simulations}

To aid with the interpretation of our results, simulations of the experiment were carried out using MuMax3 [28], a micromagnetic simulation software package. Physical parameters were set such that saturation magnetisation, $M_{\text {sat }}=197 \mathrm{kA} / \mathrm{m}$, exchange stiffness, $A_{\text {ex }}=3.5 \times 10^{-12} \mathrm{~J} / \mathrm{m}$, and Gilbert damping, $\alpha=5 \times 10^{-5}$ [6]. As in the experiment, the probe antenna was $3 \mathrm{~mm}$ from the pumps, while a separate detection region was defined $1 \mathrm{~mm}$ from the pumped region. The pumps were driven at $3.915 \mathrm{GHz}$ and the probe driven at $3.91825 \mathrm{GHz}$ with each field configuration simulated for $2 \mu$ s before performing a Fourier transform to investigate the frequency response of each system.

The secondary effects caused by reflections of spin waves from the short ends of the waveguide were controlled for by increasing the value for $\alpha$ in these regions by factor of 300 . The regions of waveguide edge directly in contact with the pump antennae were also assigned the increased damping value. By suppressing these edgereflections, any output signals may be attributed purely to the nonlinear interaction of the excited pump and probe magnons.

Figure 6 shows amplitudes of pump and probe magnons for different magnetic fields. The white regions represent the simulated waveguides, while the red and blue represent the amplitude of the $x$ component of the transverse magnetisation, $m$. In the simulations, the probe magnons are launched from an antenna placed at the leftmost edge of the waveguide. At every point along the waveguide, the magnons excited at $H_{1}=30510$ e are weaker than those excited at $\mathrm{H}_{2}=3082 \mathrm{Oe}$. It is clear from the many more nodes across the width of the waveguide that (a) shows a higher width mode than in (c), and that its propagation efficiency is reduced. This phenomenon manifests itself 


\begin{tabular}{|c|c|c|c|c|c|}
\hline & & $f_{\mathrm{p}}$ - probe magnons & & p magnons & \multirow{3}{*}{0} \\
\hline \multirow{2}{*}{ 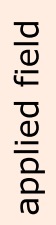 } & $H_{1}$ & (a) & (b) & 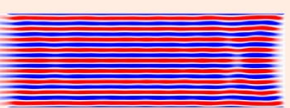 & \\
\hline & $H_{2}$ & 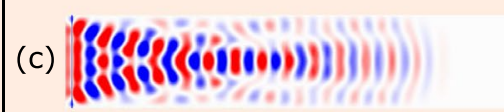 & (d) & & \\
\hline
\end{tabular}

Fig. 6 Simulations. Examples of $m_{x}$ amplitudes for applied magnetic field $\mathrm{H}=3051 \mathrm{Oe}$ and 3082 Oe. The images isolate the different amplitudes of magnons with $f_{\mathrm{p}}=3.91825 \mathrm{GHz}$ and $f_{1}=3.915 \mathrm{GHz}$. Subfigures a and c compare how transmission effi-

experimentally as highlighted in Fig. 5, where a reduced phase conjugate signal is evident at $H_{1}$ compared to $H_{2}$.

The simulation also illuminated the pump behaviour. Figure $6 \mathrm{~b}$ and $\mathrm{d}$ both shows a standing spin wave across the width of the waveguide. At lower field, there are 18 nodes across the width, compared to the 12 nodes present at $H_{2}$. As the field increases from $H_{1}$ to $H_{2}$, the intensity of the standing wave will oscillate with every node that is removed. Given Eq. (4) this phenomenon explains the 5 Oe oscillation in Fig. 5, with the simulation matching the experiment well. Indeed, increasing from $H_{1}$ to $H_{2}$ the calculation shows a difference of 6 nodes, while for the same measured fields, the intensity goes through 6 oscillations.

A time-domain Fourier transform was performed on the simulated data. A typical example is shown in Fig. $3 \mathrm{c}$ where the applied field is 3077 Oe as it was for the data measured in (b). The large peak at $f_{\mathrm{p}}$ is due to the detection region being placed between the probe and pump antennae, therefore picking up the original probe signal. The width of this peak is an artefact of both the limited computational resolution and the conversion to a logarithmic scale. Also of note is the comparatively small power of the pump frequency at $f_{1}$ which is due to leakage of pump magnons, which are generally well confined between the antennae as shown in Fig. $6 \mathrm{~b}$ and $\mathrm{d}$. We also observe a small bump at $3.925 \mathrm{GHz}$ which arises from a higher order mixing term.

The significance of Figs. $3 \mathrm{~b}$ and $\mathrm{c}$ is a prominent peak at $f_{\mathrm{c}}$ in both simulation and measurement. Because the simulated system eliminates reflections from the ends of the waveguide, this signal must necessarily be reflecting from the pumped region of periodic potential. Since this signal was generated by FWM and has angular frequency $\omega_{\mathrm{c}}=\omega_{1}+\omega_{2}-\omega_{\mathrm{p}}$, it must also have wavevector $\mathbf{k}_{\mathrm{c}}$ in order to satisfy equation (4) confirming that it is indeed a phase conjugate of the probe signal. Furthermore, the absence of the peak at $2 f_{\mathrm{p}}-f_{1}$ in the simulated spectrum supports the notion that it was due to a third-order nonlinear effect caused by reflections from the waveguide edge. ciency of probe magnons is affected by specific width modes. Both b and $\mathbf{d}$ show a standing wave created by the pump antennae. The standing wave at field $H_{1}$ has 18 nodes, compared to the 12 nodes at $\mathrm{H}_{2}$

\section{Conclusion}

In summary, this work demonstrates through experiments and simulations the generation of a phase conjugate magnon from a non-degenerate four-wave mixing process. The phase conjugate signal is enhanced when the applied magnetic field strength is such that the pumps form a standing wave across the width of the waveguide. This standing wave causes a large pump amplitude which significantly increases the nonlinearity of region. The geometry of the experiment and simulation ensure that any return signal at $f_{c}$ must be a phase conjugate signal.

This new phase conjugate differs from previous observations in magnonic systems in three ways: (1) It utilises CW signals rather than spin wave bullets or pulsed signals (2) It uses a third-order FWM process in contrast to threewave parametric pumping. (3) We utilise the 2D nature of the waveguide with perpendicularly travelling spin waves. Future work would involve the creation of degenerate FWM, though discriminating between monochromatic signals poses different challenges. Our work opens the door for this type of phase conjugation as yet another process that is exploitable in novel magnon-based computational paradigms.

Acknowledgements We would like to extend our gratitude towards Prof. Paul Ewart for his invaluable insight into the nuances of this experiment. This research was partially funded by Magdalen College, Oxford.

\section{Compliance with ethical standards}

Conflict of interest On behalf of all authors, the corresponding author states that there is no conflict of interest.

Open Access This article is distributed under the terms of the Creative Commons Attribution 4.0 International License (http://creat ivecommons.org/licenses/by/4.0/), which permits unrestricted use, distribution, and reproduction in any medium, provided you give appropriate credit to the original author(s) and the source, provide 
a link to the Creative Commons license, and indicate if changes were made.

\section{References}

1. Archilla JF, Palmero F, Lemos MC, Sanchez-Rey B, Casado-Pascual $J$ (2018) Nonlinear systems. Understanding complex systems, vol 2. Springer, Cham. https://doi.org/10.1007/978-3-319-72218 $-4$

2. Bloom DM, Bjorklund GC (1977) Conjugate wavefront generation and image reconstruction by four wave mixing. Appl Phys Lett 31(9):592-594. https://doi.org/10.1063/1.89791

3. Boyd RW (2008) Nonlinear optics, 3rd edn. Academic Press Inc., Orlando

4. Chiou AE (1999) Photorefractive phase-conjugate optics for image processing, trapping, and manipulation of microscopic objects. Proc IEEE 87(12):2074-2085. https://doi. org/10.1109/5.805922

5. Chumak AV, Vasyuchka V, Serga AA, Hillebrands B (2015) Magnon spintronics. Nat Phys 11(June):453-461. https://doi. org/10.1038/NPHYS3347

6. Chumak AV, Serga AA, Hillebrands B (2017) Magnonic crystals for data processing. J Phys D Appl Phys 50(24):244001. https:// doi.org/10.1088/1361-6463/aa6a65

7. Cornelissen LJ, Liu J, Van Wees BJ, Duine RA (2018) Spin-currentcontrolled modulation of the magnon spin conductance in a three-terminal magnon rransistor. Phys Rev Lett. https://doi. org/10.1103/PhysRevLett.120.097702

8. Csaba G, Papp Á, Porod W (2017) Perspectives of using spin waves for computing and signal processing. Phys Lett A 381(17):1471-1476. https://doi.org/10.1016/j.physl eta.2017.02.042

9. Ewart P, O'Leary SV (1986) Detection of $\mathrm{OH}$ in a flame by degenerate four-wave mixing. Opt Lett 11(5):279. https://doi. org/10.1364/OL.11.000279

10. Fisher RA, Suydam BR, Yevick D (1983) Optical phase conjugation for time-domain undoing of dispersive self-phase-modulation effects. Opt Lett. https://doi.org/10.1364/OL.8.000611

11. Gruszecki P, Kasprzak M, Serebryannikov AE, Krawczyk M, Smigaj W (2016) Microwave excitation of spin wave beams in thin ferromagnetic films. Sci Rep https://doi.org/10.1038/srep22367. arXiv:1509.05061

12. Haldar A, Tian C, Adeyeye AO (2017) Isotropic transmission of magnon spin information without a magnetic field. Sci Adv. https://doi.org/10.1126/sciadv.1700638

13. Kalinikos B, Kovshikov N, Kostylev M, Benner H (1998) Parametric frequency conversion with amplification of a weak spin wave in a ferrite film. IEEE Trans Magn 34(4):1393-1395. https://doi. org/10.1109/20.706559

14. Kalinikos BA, Slavin AN (1986) Theory of dipole-exchange spin wave spectrum for ferromagnetic films with mixed exchange boundary conditions. J Phys C Solid State Phys 19(35):70137033. https://doi.org/10.1088/0022-3719/19/35/014

15. Kazakov GT, Kozhevnikov AV, Filimonov YA (1997) Fourmagnon decay of magnetostatic surface waves in yttrium iron garnet films. Phys Solid State 39(2):288-295. https://doi. org/10.1134/1.1129801

16. Khivintsev Y, Marsh J, Zagorodnii V, Harward I, Lovejoy J, Krivosik P, Camley RE, Celinski Z (2011) Nonlinear amplification and mixing of spin waves in a microstrip geometry with metallic ferromagnets. Appl Phys Lett doi 10(1063/1):3541787

17. Li C, Jiang Z, Zhang Y, Zhang Z, Wen F, Chen H, Zhang Y, Xiao M (2017) Phys Rev Appl 7(1):014023. https://doi.org/10.1103/ PhysRevApplied.7.014023
18. Manipatruni S, Nikonov DE, Young IA (2018) Beyond CMOS computing with spin and polarization. Nat Phys. https://doi. org/10.1038/s41567-018-0101-4

19. Marsh J, Camley RE (2012) Two-wave mixing in nonlinear magnetization dynamics: a perturbation expansion of the LandauLifshitz-Gilbert equation. Phys Rev B Condens Matter Mater Phys 86(22):1-7. https://doi.org/10.1103/PhysRevB.86.224405

20. Melkov GA, Vasyuchka VI, Chumak AV, Slavin AN (2005) Doublewave-front reversal of dipole-exchange spin waves in yttriumiron garnet films. J Appl Phys 10(1063/1):2077842

21. Melkov GA, Koblyanskiy YV, Slipets RA, Talalaevskij AV, Slavin AN (2009) Nonlinear interactions of spin waves with parametric pumping in permalloy metal films. Phys Rev B Condens Matter Mater Phys 79(13):1-9. https://doi.org/10.1103/PhysR evB.79.134411

22. Pepper DM, Abrams RL (1978) Narrow optical bandpass filter via nearly degenerate four-wave mixing. Opt Lett 3(6):212-214

23. Prabhakar A, Stancil DD (2009) Spin waves. Springer US, Boston. https://doi.org/10.1007/978-0-387-77865-5. arXiv:1011.1669v3

24. Sadovnikov AV, Odintsov SA, Beginin EN, Grachev AA, Gubanov VA, Sheshukova SE, Sharaevskii YP, Nikitov SA (2018) Nonlinear spin wave effects in the system of lateral magnonic structures. JETP Lett. https://doi.org/10.1134/S0021364018010113

25. Serga AA, Hillebrands B, Deraokritov SO, Slavin AN, Wierzbicki P, Vasyuchka V, Dzyapko O, Chumak A (2005) Parametric generation of forward and phase-conjugated spin-wave bullets in magnetic films. Phys Rev Lett. https://doi.org/10.1103/PhysR evLett.94.167202

26. Stigloher J, Decker M, Körner HS, Tanabe K, Moriyama T, Taniguchi T, Hata H, Madami M, Gubbiotti G, Kobayashi K, Ono T, Back CH (2016) Snell's law for spin waves. Phys Rev Lett. https://doi. org/10.1103/PhysRevLett.117.037204. arXiv:1606.02895

27. Unnikrishnan G, Joseph J, Singh K (2008) Optical encryption system that uses phase conjugation in a photorefractive crystal. Appl Opt. https://doi.org/10.1364/ao.37.008181

28. Vansteenkiste A, Leliaert J, Dvornik M, Helsen M, Garcia-Sanchez F, Van Waeyenberge B (2014) The design and verification of MuMax3. AIP Adv 10(1063/1):4899186. arXiv:1406.7635

29. Waldrop MM (2016) The chips are down for Moore's law. Nature 530(7589):144-147. https://doi.org/10.1038/530144a

30. Wu Z, Zhang Y, Yuan C, Wen F, Zheng H, Zhang Y, Xiao M (2013) Cubic-quintic condensate solitons in four-wave mixing. Phys Rev A At Mol Opt Phys. https://doi.org/10.1103/PhysRevA.88.06382 8

31. Yariv A, Pepper DM (1977) Amplified reflection, phase conjugation, and oscillation in degenerate four-wave mixing. Opt Lett. https://doi.org/10.1364/OL.1.000016

32. Zhang Y, Nie Z, Zhao Y, Li C, Wang R, Si J, Xiao M (2010) Modulated vortex solitons of four-wave mixing. Opt Express. https:// doi.org/10.1364/oe.18.010963

33. Zhang Y, Wang Z, Nie Z, Li C, Chen H, Lu K, Xiao M (2011) Fourwave mixing dipole soliton in laser-induced atomic gratings. Phys Rev Lett 106(9):093904. https://doi.org/10.1103/PhysR evLett.106.093904

34. Zografos O, Soree B, Vaysset A, Cosemans S, Amaru L, Gaillardon PE, De Micheli G, Lauwereins R, Sayan S, Raghavan P, Radu IP, Thean A (2016) Design and benchmarking of hybrid CMOS-spin wave device circuits compared to $10 \mathrm{~nm}$ CMOS. In: IEEE-NANO 2015: 15th international conference on nanotechnology, pp 686-689. https://doi.org/10.1109/NANO.2015.7388699

Publisher's Note Springer Nature remains neutral with regard to jurisdictional claims in published maps and institutional affiliations. 